\title{
Determination of diagnostic value (validity) leukocyte esterase (urine dipstick strip) in differentiating inflammatory arthritis from bacterial arthritis
}

Mehrnoush Hassas Yeganeh ${ }^{1} \mathbb{D}$, Maryam Talaei ${ }^{2} \mathbb{D}$, Alireza Ebrahimi Bazzaz ${ }^{2} \mathbb{D}$, Khosro Rahmani² Keza $^{2}$ Sinaei $^{3} \mathbb{D}$, Mohamadreza Fathi ${ }^{4}$ (D) Reza Shiari ${ }^{2}$ (D) and Hamid Hosseinzadeh ${ }^{5^{*}}$ (D)

\begin{abstract}
Background: The current diagnostic cornerstone for septic arthritis contains gram stains, bacterial culture, and cell count with a differential of aspirated synovial fluid. Recently, a synovial leukocyte esterase (LE) test has been used for diagnosing septic arthritis. Since this test measures the esterase activity of leukocytes, there is always a dilemma for using this test in patients with inflammatory arthritis.

Methods: We collected the synovial fluid specimens as part of the general diagnostic protocol for patients suspected of Juvenile Idiopathic Arthritis (JIA) or Septic Arthritis (SA). Each group included 34 patients. We compared the result of the synovial LE test with the result of the culture of each patient.

Results: The mean ages of patients were $64.14 \pm 31.27$ and $50.88 \pm 23.19$ months in the JIA group and septic arthritis group, respectively. The LE test results were positive in 30 specimens, trace in 3 and negative in one in the first-time test and were positive in 31 specimens and trace in 3 in the second-time test, while it was negative in all patients with JIA. Hence, the sensitivity of the synovial LE test was $80.8 \%$, the specificity, PPV, and NPV were 78.6, 70.0, 86.8\% respectively based on a positive culture.

Conclusion: The leukocyte esterase strip test can be used as a rapid, bedside method for diagnosing or excluding bacterial infections in different body fluids. The synovial LE test can be used as an accurate test to rapidly rule in or out an acute articular bacterial infection, even in patients with concurrent inflammatory arthritis.
\end{abstract}

Keywords: Bacterial arthritis, Septic, Leukocyte esterase, Inflammatory arthritis, Synovial white cell count

\section{Background}

Septic arthritis is a joint infection that can lead to significant acute and chronic morbidities. The overall incidence of this condition is 4 to 10 per 100,000 children in developed countries [1] and up to 200 per 100,000 in developing countries [2]. More than $80 \%$ of cases occur in lower extremities, knees, hips and ankles being the most commonly affected joints [3]. The male to female ratio is 1.4/1 [3]. An infection is acute if the time between symptom onset and diagnosis is $<2$ weeks [2]. If

\footnotetext{
* Correspondence: Hamid@rowan.edu

${ }^{5}$ Orthopedic Research Group, School of Osteopathic Medicine, Rowan

University, 201 S Broadway, Camden, NJ 08103, USA

Full list of author information is available at the end of the article
}

diagnosed late, acute bacterial septic arthritis can lead to chondral damage (as early as $8 \mathrm{~h}$ ) and joint destruction (permanent joint damage can occur in $<3$ days), and adjacent bone osteomyelitis [1], hence, urgent diagnosis and treatment are warranted. Diagnosis of acute bacterial arthritis depends on clinical presentations, lab findings, imaging studies and arthrocentesis. However, none of these parameters are specific and sensitive enough to rule in or out the acute bacterial arthritis [4]. The cornerstone of the diagnosis is the evaluation of aspirated synovial fluid by gram stain, bacterial culture, and cell count with differential [1].

Recently, new diagnostic markers have been investigated, including synovial leukocyte esterase $[5,6]$, 
synovial alpha-defensin [7], serum interleukine-6 [8], serum procalcitonin [3], serum D-dimer [9] and molecular technologies [8]. Synovial leukocyte esterase (LE) can be measured with a colorimetric strip (urinalysis dipstick), which is a quick, easy, simple and inexpensive test, providing immediate test results, being invaluable in the operative setting. Synovial LE has a sensitivity of $81 \%$ and specificity of $97 \%$ using a 2-plus $(++)$ reading as a threshold for bacterial arthritis [10]. LE test has been proved accurate for diagnosis of meningitis [11], peritonitis [12] and even cow mastitis [13]. Since the LE test shows elevated WBC count in the tested fluid, there is no study in children assessing the effect of inflammatory WBC on the LE test results. So, based on the current literature, we cannot differentiate between inflammatory arthritis with elevated synovial WBC count and acute bacterial arthritis, which may be very similar clinically in children, solely on the result of the synovial LE test. We designed this study to evaluate the diagnostic value of synovial LE test in the acute bacterial arthritis in children and differentiating them from inflammatory arthritides.

\section{Material and methods}

Research method, community, sample and sampling method

We measured the minimum acceptable sample size with Cochrane Formula (Formula 1)

$$
n_{0}=\frac{Z^{2} p q}{\mathrm{e}^{2}}
$$

Where, e is the desired level of precision, which we considered 0.1, $\mathrm{p}$ is the (estimated) proportion of the population which has the attribute in question, which is $87 \%$ based on previous studies, $\mathrm{q}$ is $1-\mathrm{p}$ and $\mathrm{Z}$ is derived from $Z$ Table, which is 1.96 . Hence, the minimum necessary sample size is 43 .

$$
(1.96 \times 1.96)(0.87)(0.13) /(0.1 \times 0.1)=43.44
$$

We evaluated the synovial fluid specimens collected as part of the general diagnostic protocol from patients suspected of Juvenile Idiopathic arthritis or Septic Arthritis, during a period between December 2014 and April 2016 in pediatric rheumatology department at Mofid's children hospital and orthopedic department at Akhtar Orthopedic Hospital.

JIA was diagnosed based on ILAR criteria of JIA, 2001 [14]:

- Systemic arthritis was diagnosed if there was arthritis in 1 or more joints with or preceded by fever of at least two weeks' duration. Signs or symptoms must have been documented daily for at least three days and accompanied by one or more of the following: evanescent rash, generalized lymphadenopathy, hepato/splenomegaly, serositis.

- Oligoarthritis was diagnosed if there is arthritis affecting 1 to 4 joints during the first six months.

- The diagnosis of septic arthritis was confirmed by synovial WBC count $>50,000 / \mathrm{mL}$ and positive synovial fluid culture.

Joint aspiration in JIA patients was performed by a pediatric rheumatologist and in septic arthritis patients was performed by an orthopedic surgeon. We included 34 patients diagnosed with JIA and 34 patients diagnosed with septic arthritis in this investigation. All synovial fluid specimens from patients with JIA and septic arthritis were evaluated for the LE test. For the LE test, the synovial fluid specimens were collected in dry tubes and tested within $30 \mathrm{~min}$ using Accutest ${ }^{\circ} \mathrm{URS}-10 \mathrm{~T}$ strips (Accutest, Ca, USA). LE test was read after $120 \mathrm{~s}$ (according to the strip directions) and considered positive if the result was 2-plus (++) (Fig. 1). The reproducibility of the LE test was assessed by performing two tests a few minutes apart and read by two different researchers. Same day microscopic assessment and culture were performed on all synovial fluid samples.

The study protocol was approved by the ethical committee of the hospital.

\section{Statistics}

Sensitivity (Se), specificity (Sp), positive predictive value (PPV) and negative predictive value (NPV) were determined using the synovial culture result as the standard. Means were compared using the Kruskal-Wallis test and percentages using the Chi-square test. A Cohen's $\mathrm{k}$ test was run to determine if there was an agreement between the result of culture and LE tests. All these analyses were performed by SPSS, version 22 (IBM, NY, USA). Pvalues smaller than 0.05 were considered significant.

\section{Results}

The mean age in the JIA group was $64.14 \pm 31.27$ months (26-130). Eighteen patients were male, and 16 were female $(\mathrm{M} / \mathrm{F}$ ratio 1.125$)$. In the septic arthritis group, the mean age was $50.88 \pm 23.19$ months (11-89). Twenty-one patients were male and 13 were female (M/ F ratio 1.615).

The main sources for synovial fluid specimens in septic arthritis group were knee (52.94\%), hip (23.53\%) and ankle $(23.53 \%)$ and in JIA were knee $(55.89 \%)$, ankle (32.35\%), elbow (8.82\%) and wrist (2.94\%).

In the JIA group, mean WBC count was $31,811 \pm 9425$ $(18,500->50,000)$, with the PMN mean percentage of $64.70 \pm 8.28$ (49-87). The LE test results in all specimens were negative $(100 \%)$ in the first-time test and were 


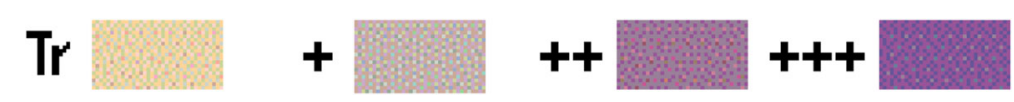

Fig. 1 title: How to diagnose an infection using the Dipstick bar. $\operatorname{Tr}=\operatorname{Trace},(+)=$ One plus, $(++)=$ two plus, $(+++)=\operatorname{tree}$ plus. $\operatorname{Tr}$ and $(+)$ are considered as negative results. $(++)$ and $(+++)$ are considered as positive results

negative in 33 specimens and trace $(+)$ in one in the second-time test.

In the septic arthritis group, mean WBC count was 43, $261 \pm 8656(23,000->50,000)$, with the PMN mean percentage of $84.73 \pm 5.88$ (75-94). The culture result was S. aureus (27 cases [79.41\%]), S. epidermidis (2 cases [5.88\%]), N. gonorrhea (2 cases [5.88\%]) and polymicrobial (3 cases $[8.82 \%])$. The LE test results were positive $(++)$ in 30 specimens $(88.23 \%)$, trace $(+)$ in $3(8.82 \%)$ and negative in one $(2.94 \%)$ in the first-time test and were positive $(+++$ or ++$)$ in 31 specimens $(91.17 \%)$ and trace $(+)$ in $3(8.82 \%)$ in the second-time test. The reproducibility of the synovial LE test was assessed by testing all the specimens twice, which showed a 76.9\% reproducibility.

Based on the positive culture, the sensitivity of the synovial LE test is $80.8 \%$, the specificity is $78.6 \%$, PPV is $70.0 \%$ and NPV is $86.8 \%$ (Table 1). Cohen's $\mathrm{K}$ demonstrated statistically a significant moderate agreement between the results specimen's culture and the LE test, $\mathrm{K}=.577$ (95\% CI), $p<.0005$ (Table 2).

Based on the synovial WBC count as a marker for joint infection, the sensitivity of the synovial LE test is $83.3 \%$, the specificity is $70.0 \%$, PPV is $50.0 \%$ and NPV is 92.1\% (Table 3).

\section{Discussion}

Prompt diagnosis of bacterial infection of the synovial joints is of paramount importance [15]. There are several criteria to make this diagnosis; however, each having some shortcomings. Clinical manifestations and blood test findings can only guide to suspicion of, not making, the diagnosis [16]. No single investigation has 100\% sensitivity for the diagnosis of acute bacterial arthritis. Synovial fluid WBC count $(>50,000 / \mathrm{mL}$ versus $<50,000 /$ $\mathrm{mL}$ ) is either not sufficiently reliable to rule in or out a diagnosis of acute bacterial arthritis [17, 18]. Synovial fluid PMN percent $\geq 90 \%$ has a sensitivity of $73 \%$ and specificity of $79 \%$ for acute bacterial arthritis [19]. Synovial fluid gram staining is positive in only $50 \%$ of cases [17]. The most specific test is recovering the bacteria

Table 1 The efficiency of synovial LE test compared with the results of synovial fluid culture

\begin{tabular}{lccll}
\hline Diagnostic test & Sensitivity & Specificity & PPV & NPV \\
\hline Synovial LE & $80.8 \%$ & $78.6 \%$ & $70.0 \%$ & $86.8 \%$ \\
\hline
\end{tabular}

PPV $=$ Positive Predictive Value. NPV = Negative Predictive Value. $\mathrm{LE}=$ leukocyte esterase from the synovial fluid by culture, but it is not a sensitive test and has many false-negative results [16]. Besides, it is a time-consuming test, which can delay the diagnosis in controversial cases, increasing the likelihood of joint destruction [1]. There is increasing evidence in favor of the measurement of some markers in serum and synovial fluid for diagnosing acute bacterial arthritis. Serum interleukin- 6 has shown a sensitivity of $97 \%$ and specificity of $91 \%$ [8], synovial fluid $\alpha$-defensin showed $100 \%$ specificity and sensitivity, although the number of studies is limited [10], serum D-dimer $>850 \mathrm{ng} / \mathrm{mL}$ has a specificity of $89 \%$ and specificity of $93 \%$ for diagnosis of bacterial arthritis [9].

Tarabichi et al. reported that the synovial LE strip test has a sensitivity of $100 \%$ and specificity of $83.33 \%$ with PPV 95.45\% and NPV 100\% in diagnosing bacterial arthritis around the joint prostheses, especially if associated with abnormal serology (Elevated ESR and CRP) [20]. Gautam et al. showed a sensitivity of $79.2 \%$, a specificity of $80.8 \%$, PPV of $61.8 \%$ and NPV of $90.1 \%$ for diagnosing acute bacterial arthritis in native joints [5]. Colvin et al. showed less sensitivity and specificity and PPV, but a $100 \%$ NPV for synovial LE test in native joints [21].

This test has been used as a bedside test in the diagnosis of urinary tract infections since the 1980s and proved accurate in the diagnosis of infection in bacterial meningitis, bacterial peritonitis, middle ear effusion and pleural infections.

Kolhe et al. showed LE test to be a useful approach for diagnosis of subacute bacterial peritonitis, with a sensitivity of $100 \%$, a specificity of $94 \%$, PPV of $57 \%$ and NPV of $94 \%$ [12].

Lebovics et al. reported promising accuracy for the diagnosis of chronic middle ear infection [22]. Koulaouzidis et al. in a systematic review on the accuracy of LE test in the diagnosis of spontaneous bacterial peritonitis, reported a sensitivity ranging from 45 to $100 \%$, specificity ranging from 81 to $100 \%$, PPV ranging from 42 to $100 \%$ and NPV ranging from 87 to $100 \%$ [23].

Bortcosh et al. in a meta-analysis of utility of LE test in the diagnosis of meningitis, showed a sensitivity of $92 \%$, specificity of $98 \%$ and NPV of $99 \%$ [11].

As literature shows, the synovial LE test has proved to be a sensitive, specific, low-cost $(<1 \$)$ and very rapid $(<$ $3 \mathrm{~min}$ ) test for rapid bedside ruling in or out the infection.

When LE test is used for diagnosing acute bacterial synovitis, since it is dependent on the leukocytes, there 
Table 2 Cohen's kappa measures showed the agreement between the results of culture and LE test

\begin{tabular}{llllll}
\hline Symmetric Measures & & Value & Asymptotic Standard Error & Approximate T & $P$ value \\
\hline Measure of Agreement & Kappa & .577 & .1 & 4.789 & .000 \\
\hline
\end{tabular}

$P$-value $<0.0005$

is always a special dilemma to differentiate between acute bacterial arthritis and chronic inflammatory arthritis, both of which are associated with a very high number of WBCs with a high percentage of PMNs in the synovial fluid.

Coiffier et al. compared the result of the synovial LE test in patients with inflammatory (including septic and rheumatoid arthritides) versus non-inflammatory arthritis (mechanical arthritis). This study showed that the synovial LE test could accurately differentiate between inflammatory arthritis with synovial WBC $>2000 / \mathrm{mm}^{3}$ and non-inflammatory arthritis with synovial WBC < $2000 / \mathrm{mm}^{3}$. However, they did not assess the ability of the synovial LE test to differentiate between septic and rheumatoid arthritis [24].

Since inflammatory arthritis is a risk factor for septic arthritis and sometimes their clinical presentations overlap, rapid differentiation between inflammatory and septic arthritis may not be feasible. Our study is the first to report a head-to-head comparison of the result of the synovial LE test in patients with confirmed JIA and septic arthritis to find a rapid method for differentiation. Our results show that the synovial LE test was positive in the majority of patients who had positive synovial culture (91.17\%); however, interestingly, this test was negative in all patients with confirmed JIA based on ILAR criteria and negative synovial fluid culture. The mean synovial WBC count was $31,811 \pm 9425(18,500->50$, $000)$ in JIA group and 43,261 $\pm 8656(23,000->50,000)$ in septic arthritis group. The synovial LE test was negative even in JIA patients who had synovial WBC count in the septic range $(>50,000)$. In all these specimens, the PMN percentage was between 49 and $94 \%$. We speculate that the leukocytes need a bacterial presence for their activity and leukocyte esterase production. Hence, when there are no bacteria, the leukocytes are not activated and will no produce leukocyte esterase, even when leukocytes are present in large numbers. This theory needs to be proved; however, the result of this study shows that we can use a synovial LE test to diagnose or exclude acute bacterial arthritis, even in patients with

Table 3 The efficiency of synovial LE test compared with the results of synovial WBC count

\begin{tabular}{lcccc}
\hline Diagnostic test & Sensitivity & Specificity & PPV & NPV \\
\hline Synovial LE & $83.3 \%$ & $70.0 \%$ & $50.0 \%$ & $92.1 \%$
\end{tabular}

PV $=$ Positive Predictive Value. NPV = Negative Predictive Value. $\mathrm{LE}=$ leukocyte esterase inflammatory arthritis with a huge number of WBC in synovial fluid.

This study has some limitations. The first limitation is the relatively small number of patients in both groups. We need to perform a multicenter study will larger numbers of patients to get more reliable results. The second limitation is the manual reading of the result of the LE test, which decreases the accuracy of strip reading.

Conclusion Absence of positive reaction in JIA, helps us to use Leukocyte esterase strip test as a rapid, bedside method for differentiating a septic arthritis from an actively inflamed joint in JIA. Based on our study, the synovial LE test is an accurate test to rapidly rule in or out an acute articular bacterial infection, even in patients with concurrent inflammatory arthritis.

\section{Abbreviations \\ JIA: Juvenile Idiopathic arthritis; LE: Leukocyte esterase; \\ PMN: Polymorphonuclear; SA: Septic Arthritis; WBC: White Blood Cell}

\section{Acknowledgements \\ Is not applicable.}

\section{Authors' contributions}

MHY designed the study and performed Whole research supervision and conduct, Major contribution to manuscript preparation. MT and AEB worked on the medical Thesis, data collection and analysis, manuscript preparation. $\mathrm{KR}, \mathrm{RS}$, and RS Substantially revised the manuscript. HS worked on the Drafting the article and revising it critically for important intellectual content. All authors reviewed and final approval and approved the submitted version of the manuscript.

\section{Funding}

This research did not receive any specific grant from funding agencies in the public, commercial, or not-for-profit sectors.

\section{Availability of data and materials}

The datasets analyzed during the current study are available from the corresponding author on reasonable request.

\section{Ethics approval and consent to participate}

Before undergoing any procedures, all parents of the patients who were included in the present study signed an informed consent form approved by the research ethics committee at the Shahid Beheshti Medical University (SBMU). We have obtained ethical approval, according to ethical standards of the local committee of the Shahid Beheshti Medical University (SBMU)'s ethics board and with the Declaration of Helsinki. The ethical approval number: IR.SBMU.RETECH.REC.1395.158.

Consent for publication

Not applicable.

Competing interests

The authors declare that they have no competing interests. 


\section{Author details}

${ }^{1}$ Mofid Clinical Research Development Center, SBMU, Tehran, Iran. ${ }^{2}$ Mofid Children Hospital, SBMU, Tehran, Iran. ${ }^{3}$ Kerman Medical University, Kerman, Iran. ${ }^{4}$ Jondi Shapour Medical University, Ahvaz, Iran. ${ }^{5}$ Orthopedic Research Group, School of Osteopathic Medicine, Rowan University, 201 S Broadway, Camden, NJ 08103, USA.

Received: 9 May 2019 Accepted: 19 January 2020

Published online: 28 January 2020

\section{References}

1. Montgomery NI, Epps HR. Pediatric septic arthritis. Orthop Clin North Am. 2017:48(2):209-16.

2. Iliadis $A D$, Ramachandran M. Paediatric bone and joint infection. EFORT Open Rev. 2017;2(1):7-12.

3. Arnold JC, Bradley JS. Osteoarticular infections in children. Infect Dis Clin N Am. 2015 Sep;29(3):557-74.

4. Dartnell J, Ramachandran M, Katchburian M. Haematogenous acute and subacute pediatric osteomyelitis. J Bone Joint Surg Br. 2012;94-B(5):584-95.

5. Gautam V, Saini R, Sharma S. Effectiveness of leucocyte esterase as a diagnostic test for acute septic arthritis. J Orthop Surg. 2017;25(1): 230949901668501.

6. Kelly EG, Cashman JP. Leucocyte esterase in the rapid diagnosis of paediatric septic arthritis. Med Hypotheses. 2013;80(2):191-3.

7. Deirmengian C, Kardos K, Kilmartin P, Cameron A, Schiller K, Booth RE, et al. The alpha-defensin test for Periprosthetic joint infection outperforms the leukocyte esterase test strip. Clin Orthop Relat Res. 2015;473(1):198-203.

8. Goswami K, Parvizi J, Maxwell CP. Current recommendations for the diagnosis of acute and chronic PJI for hip and knee-cell counts, alphaDefensin, leukocyte esterase. Next-generation Sequencing Curr Rev Musculoskelet Med. 2018;11(3):428-38.

9. Shahi A, Kheir MM, Tarabichi M, Hosseinzadeh HRS, Tan TL, Parvizi J. Serum D-dimer test is promising for the diagnosis of Periprosthetic joint infection and timing of Reimplantation. J Bone Jt Surg - Am Vol. 2017;99(17):1419-27.

10. Wyatt MC, Beswick AD, Kunutsor SK, Wilson MJ, Whitehouse MR, Blom AW. The alpha-Defensin immunoassay and leukocyte esterase colorimetric strip test for the diagnosis of Periprosthetic infection. J Bone Jt Surg. 2016;98(12): 992-1000.

11. Bortcosh W, Siedner M, Carroll RW. Utility of the urine reagent strip leucocyte esterase assay for the diagnosis of meningitis in resource-limited settings: meta-analysis. Trop Med Int Heal. 2017;22(9):1072-80.

12. Kolhe K, Khairnar H, Chauhan S, Poddar P, Pandey V, Ingle M, et al. A study of rapid bedside diagnosis of spontaneous bacterial peritonitis using leukocyte esterase test strips. J Clin Exp Hepatol. 2018;8(July 2018):S59.

13. Mirzaei A, Khorsand A, Hajibemani A, Sharifiyazdi H. Evaluation of leukocyte esterase test strips for rapid diagnosis of subclinical mastitis in dairy cows. Comp Clin Path. 2018:1-6.

14. Eisenstein EM, Berkun Y. Diagnosis and classification of juvenile idiopathic arthritis. J Autoimmun. 2014;48-49:31-3.

15. Nusem I, Sehu M. Septic arthritis of the hip - diagnosis and management. Ann Orthop Rheumatol. 2016;4(2):1068-73.

16. Mathews CJ. Bone and joint infections. Medicine (Baltimore). 2018;46(4):24750.

17. Mathews CJ, Kingsley G, Field M, Jones A, Weston VC, Phillips M, et al. Management of septic arthritis: a systematic review. Postgrad Med J. 2008; 84(991):265-70.

18. Coutlakis PJ, Roberts WN, Wise CM. Another look at synovial fluid leukocytosis and infection. JCR J Clin Rheumatol. 2002;8(2):67-71.

19. Margaretten ME, Kohlwes J, Moore D, Bent S. Does this adult patient have septic arthritis? JAMA. 2007;297(13):1478-88.

20. Tarabichi M, Fleischman AN, Shahi A, Tian S, Parvizi J. Interpretation of Leukocyte Esterase for the Detection of Periprosthetic Joint Infection Based on Serologic Markers. J Arthroplasty. 2017;32(9):S97-S100.e1.

21. Colvin OC, Kransdorf MJ, Roberts CC, Chivers FS, Lorans R, Beauchamp CP, et al. Leukocyte esterase analysis in the diagnosis of joint infection: can we make a diagnosis using a simple urine dipstick? Skelet Radiol. 2015;44(5): 673-7.

22. Lebovics RS, Murthy W, Karmen A. Leukocyte esterase activity in effusion fluid of patients with otitis media. Otolaryngol Neck Surg. 1993; 108(3):248-50
23. Koulaouzidis A, Leontiadis Gl, Abdullah M, Moschos J, Gasem J, Tharakan J, et al. Leucocyte esterase reagent strips for the diagnosis of spontaneous bacterial peritonitis: a systematic review. Eur J Gastroenterol Hepatol. 2008; 20(11):1055-60

24. Coiffier G, Pollet S, Albert J-D, Perdriger A, Guggenbuhl P, Chales G. Usefulness and limitations of rapid urine dipstick testing for joint-fluid analysis. Prospective single-center study of 98 specimens. Jt Bone Spine. 2013;80(6):604-7.

\section{Publisher's Note}

Springer Nature remains neutral with regard to jurisdictional claims in published maps and institutional affiliations.
Ready to submit your research? Choose BMC and benefit from:

- fast, convenient online submission

- thorough peer review by experienced researchers in your field

- rapid publication on acceptance

- support for research data, including large and complex data types

- gold Open Access which fosters wider collaboration and increased citations

- maximum visibility for your research: over $100 \mathrm{M}$ website views per year

At BMC, research is always in progress.

Learn more biomedcentral.com/submissions 